\title{
Distinct Populations of Forebrain Neural Stem and Progenitor Cells Can Be Isolated Using Side-Population Analysis
}

\author{
Mina Kim and Cindi M. Morshead \\ Department of Surgery, University of Toronto, Toronto, Ontario, Canada M5S 1A8
}

\begin{abstract}
The absence of stem cell-specific markers has posed challenges to the identification and isolation of stem cells. We report the isolation of a discrete and highly enriched population of neural stem cells from clonally derived colonies of neural stem cell and progenitor cells (neurospheres) after exposure to the fluorescent DNA binding dye Hoeschst 33342 and subsequent analysis via dual wavelength flow cytometry. The low fluorescent side population comprised only $3.6 \%$ of all live cells sorted yet contained $>99 \%$ of all the neural stem cells as assayed by the formation of neurospheres in culture. Most neurosphere-derived cells are progenitor cells, and these are found within the higher fluorescence (non-side population) fraction. The isolation of a highly enriched population of self-renewing, multipotential neural stem cells was seen from both adult- and embryonic-derived neurospheres; however, the relative percentage of cells comprising the side-population and the mechanism of dye efflux varied between adult and embryonic donor tissue. Combining the side-population analysis with markers recently shown to enrich for neural stem cells afforded no further enrichment in the case of peanut agglutinin expression and size criteria; however, when the side-population analysis was combined with Lewis X (LeX) expression, a slight enrichment was seen over side-population analysis alone.
\end{abstract}

Key words: neural stem cells; FACS analysis; neurospheres; side-population; Hoechst; enrichment

\section{Introduction}

Stem cell biology in general suffers from the lack of a specific marker that unambiguously labels all stem cells and only stem cells, enabling their prospective identification. Neural stem cells are no exception. The difficulty in isolating a pure population of neural stem cells seriously limits the study of neural stem behavior and factors that regulate them. Two recent studies have described the use of antibody staining to characterize neural stem cells from the adult mouse forebrain. One study used cell size combined with a number of negative selection criteria to isolate neural stem cells (Rietze et al., 2001), and more recently expression of LeX, a carbohydrate moiety (the trisaccharide 3-Fucosyl$\mathrm{N}$-acetyllactosamine or CD15, leukocyte cluster of differentiation 15) (Gooi et al., 1981), was found to be enriched in stem cell populations (Capela and Temple, 2002). Here, we have taken advantage of the "side-population" analysis described originally to isolate a highly enriched population of hematopoietic stem cells from adult bone marrow (Goodell et al., 1996) and have shown that it is a simple and effective means by which to isolate embryonic and adult neural stem cells.

Bone marrow cells exposed to a fluorescent DNA binding dye, Hoeschst 33342, and observed using fluorescent-activated cell

Received Aug. 13, 2003; revised Sept. 29, 2003; accepted 0ct. 1, 2003.

This work was supported by operating grants to C.M.M. from the E. A. Baker Foundation and the Canadian Institutes of Health Research. We thank Derek van der Kooy for his support and insightful comments.

Correspondence should be addressed to Cindi M. Morshead, 1 King's College Circle, Room 1182, Toronto, Ontario, Canada M5S 1A8. E-mail: cindi.morshead@utoronto.ca.

Copyright $\odot 2003$ Society for Neuroscience $\quad$ 0270-6474/03/2310703-07\$15.00/0 sorting (FACS) at two emission wavelengths (red and blue) simultaneously resulted in a distinct staining profile. Two populations of cells were observed: (1) a side-population (SP; low fluorescence) and (2) a non-side-population (non-SP; higher fluorescence). The bone marrow-derived SP fraction comprised $\sim 1 \%$ of all of the total bone marrow and accounted for virtually all of the hematopietic stem cell activity using an in vivo bone marrow repopulation assay (Goodell et al., 1996). This distinct SP region was shown to be the result of differential efflux of the Hoechst dye because incubating bone marrow cells in the presence of verapamil (an inhibitor of ATP binding cassette (ABC)transporter protein activity) resulted in the complete loss of the SP fraction (Goodell et al., 1996; 1997; Storms et al., 2000).

We hypothesized that stem cells in general have an enhanced ability to pump out toxins relative to more differentiated progeny and tested this hypothesis using neural tissue. We performed an SP analysis on a mixed population of neural stem and progenitor cells derived from adult and embryonic mouse neurospheres [clonally derived colonies of cells with each single neurosphere composed of neural stem cells, progenitor cells, and no differentiated neurons and glia (Tropepe et al., 1999)] that provided a pre-enriched starting population. FACS analysis revealed two distinct populations from both embryonic and adult neurospheres: (1) SP cells that are highly enriched for neural stem cells and (2) non-SP cells that account for the vast majority of sorted cells and consist of neural progenitor cells. These findings suggest that an enhanced ability to pump out toxins is common among stem cells regardless of the tissue of origin; however, our findings 
suggest that different members of the ABC protein family mediate the dye efflux in a tissue-dependent manner. In addition, we compared the enrichment profile using the SP analysis with marker expression profiles described recently by other groups (Rietze et al., 2001; Capela and Temple, 2002) for stem cell isolation. We observed a slightly augmented enrichment within the SP profile using LeX expression that was dependent on the age of the starting population of neural stem cells (adult- or embryonicderived neurosphere cells).

\section{Materials and Methods}

Isolation and culturing of neural stem cells. Neural stem cells were isolated from embryonic day 14 forebrain germinal zones and from dissections of the adult forebrain subependyma from CD1 (Charles River) or C57BL/6 (Jackson Laboratories) mice as described previously (Tropepe et al., 1999). Bulk cultures were established ( $\sim 20$ cells per microliter), and the medium included epidermal growth factor (EGF, $20 \mathrm{ng} / \mathrm{ml}$ ) (Upstate Biotechnology, Lake Placid, NY), basic fibroblast growth factor (FGF, 10 $\mathrm{ng} / \mathrm{ml}$ ) (Upstate Biotechnology), and $1 \%$ penicillin/streptomycin (Tropepe et al., 1999). The cultures were passaged every 5-7 d as described (Tropepe et al., 1999). For single sphere passaging, each sphere was collected in a $200 \mu$ l pipette tip, transferred to a $500 \mu$ l Eppendorf tube containing $100 \mu \mathrm{l}$ of medium, triturated 40-50 times, and then transferred to a 24 -well plate in a final volume of $500 \mu \mathrm{l}$ of medium.

$S P$ cell analysis and flow cytometry. Neurospheres were collected, centrifuged at $1500 \mathrm{rpm}$ for $5 \mathrm{~min}$ at room temperature, triturated, and resuspended in the described media (in the absence of EGF and FGF) in a single-cell suspension of $1 \times 10^{6}$ cells $/ \mathrm{ml}$. Hoechst (Sigma, St. Louis, MO) was added at a final concentration of $5 \mu \mathrm{g} / \mathrm{ml}$ (embryonic cells) or $2.5 \mu \mathrm{g} / \mathrm{ml}$ (adult cells). The procedure was followed as described previously (Goodell et al., 1996, 1997). For blocking experiments, verapamil was added at $50 \mu \mathrm{m}$ final concentration (Sigma). For immunostaining, the suspension was incubated for $20 \mathrm{~min}$ at $4^{\circ} \mathrm{C}$ with FITC-conjugated PNA (1:200; Vecta) (Rietze et al., 2001) or FITC-conjugated CD15 antibody (1:200; Becton-Dickinson) and then rinsed twice with serum-free media (SFM) by centrifugation at $4^{\circ} \mathrm{C}$.

Flow cytometric sorting was conducted as described (Goodell et al., 1996, 1997) using a FACStar Plus (Becton Dickinson) or FACSDiva. The gating on forward and side scatter was not stringent. A live gate was defined on the flow cytometer using Hoechst red and blue axes to exclude dead cells and debris. Cell viability ranged from 40 to $70 \%$ during the sorting procedure. Sorting of antibody-labeled cells used FACS gates set with unlabeled cells. SP and non-SP cells were collected separately in plating medium.

SP versus non-SP cell culture. Sorted cells were plated in the presence of EGF and FGF (Tropepe et al., 1999). SP and non-SP cells were plated at equivalent densities (ranging from 1 to 10 cells per microliter depending on the numbers of cells isolated). The numbers of neurospheres were counted after 10-14 d in vitro.

Immunohistochemistry on neurospheres. Single- and double-label immunocytochemistry were performed as described previously (Tropepe et al., 1999). Briefly, single spheres were plated onto glass coverslips coated with MATRI-GEL (Becton Dickinson) in SFM containing 10\% fetal bovine serum. Five to $7 \mathrm{~d}$ later the cells were fixed with $4 \%$ paraformaldehyde and stained with antibodies to MAP2 (mouse monoclonal IgG; Boehringer Mannheim, Mannheim, Germany), glial fibrillary acidic protein antisera (rabbit polyclonal; Chemicon), and 04 (mouse monoclonal, IgM; Boehringer Mannheim). Appropriate secondary antibodies (FITC goat anti-rabbit, TRITC goat anti-mouse, dichlorotriazinyl-aminofluorescein goat anti-mouse; all from Jackson ImmunoResearch) were used.

Reverse transcription-PCR. Total RNA was isolated from equal numbers of SP and non-SP cells (4000-30,000, respectively) immediately after FACS-sort using RNeasy Mini Kit (Qiagen), precipitated in ethanol, and treated with DNase I (Invitrogen) before resuspension in $12 \mu \mathrm{l}$ of RNase-free water. Reverse transcription (RT) and PCR were performed sequentially in the same tube on equal amounts of isolated RNA (1-2 $\mu \mathrm{g}$ per sample) using the OneStep RT-PCR kit (Qiagen). The primers used for PCR amplification were as follows: ABCG2 $5^{\prime}$ end primer $5^{\prime}-\mathrm{GTC}$
AGC TGT GGA GCT GTT CGT AG, and ABCG2 3' end primer 5'-CAC AAG TGC TGT TGT CCG TTA CA; GFAP $5^{\prime}$ end primer $5^{\prime}$-GTT GTG AAG GTC TAT TCC TGG C, and GFAP $3{ }^{\prime}$ end primer 5'-TCC CTT AGC TTG GAG AGC AA. After 40 cycles of amplification, $7 \mu$ of the reaction mix was electrophoresed on $2 \%$ agarose gels and stained with ethidium bromide.

\section{Results}

Neural stem cell cultures were established from the forebrain germinal zones of embryonic and adult mice. The dissociated germinal zone cells were cultured in the presence of EGF and FGF2 to produce neurospheres, clonally derived colonies of cells each originating from a single neural stem cell. Typically, each neurosphere contains 15,000-20,000 cells, of which most are progenitor cells that have limited self-renewal capacity and more restricted lineage potential (Tropepe et al., 2000). Approximately $0.2-0.8 \%$ of neurosphere cells are neural stem cells with the ability to self renew (form new spheres after dissociation) and are multipotential (can generate neurons and glia). This range is based on the frequency of observing newly generated neurospheres after the plating of single cells from a dissociated neurosphere (range, 50-130 new spheres from a 15,000-20,000 cell sphere) (Morshead et al., 2002). Neural stem cells represent $0.2-$ $0.4 \%$ of the cells found within the adult forebrain subependyma (Morshead et al., 1998) and hence significantly less in a dissection of the whole adult forebrain. Virtually all of the cells within a neurosphere express nestin, a marker of undifferentiated cells (Lendahl et al., 1990); therefore, the use of neurosphere-derived cells as a starting population afforded us an enriched population of neural stem and progenitor cells in the absence of differentiated cell types. Neurospheres were collected from bulk cultures that could be maintained by passaging in the presence of EGF and FGF2.

\section{Neural stem cells sort to the SP}

Embryonic neurosphere-derived cells were collected from cultures passaged $<10 \times(<\mathrm{P} 10)$ and sorted on the basis of Hoechst fluorescence. Because continual passaging $(>10 \times)$ leads to cellular transformations in the cultures (Morshead et al., 2002), we kept the number of passages low enough to avoid transformations yet still be able to harvest vast numbers of cells. The analysis revealed SP and non-SP components similar to those seen with bone marrow cells (Fig. 1a,b). Samples of the SP and non-SP cells were collected separately and plated in the presence of EGF and FGF2 at equal densities (1-10 cells per microliter). The SP comprised $3.6 \pm 1.2 \%$ (Fig. $1 d$ ) of the live cells sorted [dead cells were excluded on the basis of propidium iodide (PI) uptake] but contained $98.7 \pm 21.7 \%$ of all of the neurosphere-forming cells. Thus, the frequency of isolating a stem cell is 1 in 12 SP cells and 1 in 3333 non-SP cells (Table 1). This represents a 278-fold enrichment comparing the SP and non-SP cells. Considering the frequency of stem cells within a neurosphere, the SP fraction represents a 10 - to 40 -fold increase over baseline conditions. Importantly, the SP versus non-SP fractions contain distinct populations of stem versus progenitor cells, respectively.

The adult neurosphere sorting profile revealed a smaller enrichment (7.5-fold) ( 1 of 46 SP cells and 1 of 345 non-SP cells formed neurospheres) (Fig. $1 c, d$ ) (Table 1). This may be attributable to increased death of adult stem cells after exposure to Hoechst. This hypothesis is supported by the fact that a lower Hoechst concentration $(2.5 \mathrm{vs} 5.0 \mu \mathrm{g} / \mathrm{ml})$ during sorting resulted in a significant increase in cell viability when adult-derived neurospheres were analyzed. This increased cell viability was not seen 

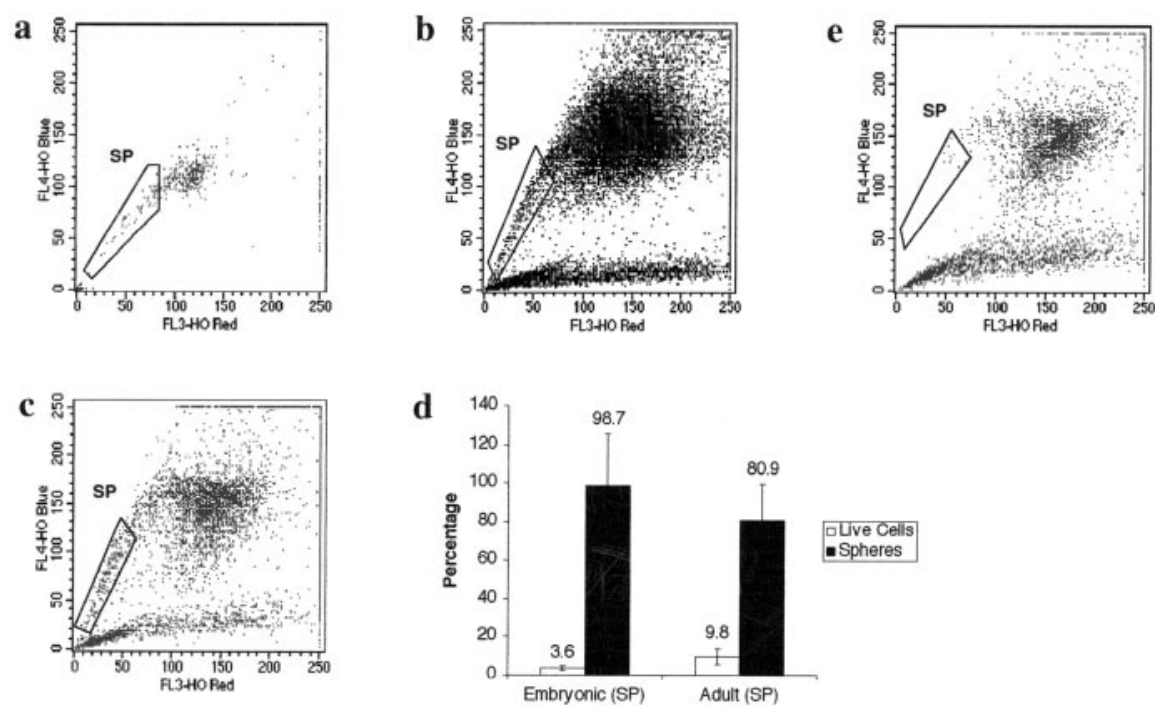

Figure 1. Neurosphere-derived cells reveal an SP that is highly enriched for neural stem cell activity. $a$, Primary mouse bone marrow cells exposed to Hoechst dye and subsequently sorted on the basis of dual wavelength flow cytometry reveal a low fluorescing SP (outlined) that comprised $1 \%$ of all of the live cells sorted. $b, c$, Neurospheres derived from embryonic $(b)$ and adult $(c)$ forebrain germinal zones were passaged $(<10 \times)$ before sorting. After exposure to Hoechst dye, SPs were observed that comprised small percentages (means \pm SEM) of all of the live cells sorted ( $d$, white bars) yet contained the vast majority of neural stem cells ( $d$, black bars; mean percentage \pm SD of all neurospheres formed from SP and non-SP cultures combined). e, Embryonic neurosphere cells were exposed to Hoechst dye and verapamil before the SP analysis. In the presence of verapamil, the SP population was completely lost (compare with $b$ ).

with embryonic cells. Alternatively, adult neural stem cells may represent a less homogenous population of cells relative to embryonic cells.

To rule out the possibility that the neural stem cell enrichment that we observed between the SP and non-SP fractions was an artifact of the toxicity of the Hoechst dye selectively killing the potentially neurosphere-forming cells in the non-SP (i.e., cells with greater levels of Hoechst dye), we performed a number of controls. The control groups consisted of (1) Hoechst only (Hoechst stained but not exposed to the FACS machine), (2) FACS machine (Hoechst stained, run through the FACS machine but not sorted into SP and non-SP), and (3) untreated (no exposure to Hoechst or FACS machine). Cells from each group were cultured at 2 cells per microliter. The number of neurospheres was counted and compared between each control group. There was a fivefold decrease in the average number of neurospheres formed after exposure to Hoechst (Hoechst only and FACS machine groups) when compared with the average number of neurospheres formed in the absence of Hoechst (untreated). There was no significant difference, however, in the average number of spheres between the Hoechst-only group and the FACS machine group (4.8 vs 4.3 neurospheres per 1000 cells plated, respectively), suggesting that although Hoechst does exert toxic effects on cells during staining, exposure to the FACS machine has no effect on the viability of these cells. More importantly, the absolute numbers of neurospheres that formed after the plating of equal numbers of sorted cells (SP plus non-SP) was virtually identical to the total numbers of neurospheres formed from Hoechsttreated cells (Hoechst only and FACS machine groups) (51.3 vs 56.8 cells formed neurospheres per 10,000 cells plated, respectively). Because the non-SP fraction contained $<0.09 \%$ of the total numbers of neurospheres that formed, virtually all of the neurosphere-forming cells are within the SP fraction. These data confirm that the SP fraction represents a true enrichment of neural stem cells.
Exposing the Hoechst-labeled neurosphere cells to verapamil, an inhibitor of the $\mathrm{ABC}$ transporter protein, resulted in a block of the formation of the SP fraction (Fig. 1e). This suggests that the distinct staining pattern observed by the neurosphere cells may be caused by a high level of dye efflux activity mediated by members of the $\mathrm{ABC}$ transporter protein family. One candidate member is Bcrp1/ABCG2, which is observed in hematopoietic stem cells (Zhou et al., 2001). To test this hypothesis, RT-PCR for Bcrp1/ABCG2 was conducted on equal amounts of RNA isolated from SP and non-SP fractions from adult- and embryonic-derived neurosphere cells (Fig. 2). The non-SP fractions (progenitor cells) from both adult and embryonic neurospheres expressed Bcrp1/ ABCG2 as well as SP cells from embryonic neurospheres. Interestingly, there was absolutely no expression from adult SP cells. Notably, although the SP fraction is enriched in neural stem cells, it also contains progenitor cells. The inability to detect Bcrp1/ABCG2 in the adult SP fraction suggests that all progenitor cells within a neurosphere are not identical and those that sort to the SP fraction are differentially expressing Bcrp1/ABCG2 compared with those that sort to the non-SP. We cannot formally rule out the possibility that Bcrp1/ABCG2 is expressed at such low levels that it is undetectable using RT-PCR; however, this seems unlikely because increasing the number of cycles of amplification did not result in Bcrp1/ABCG2 detection (data not shown). Hence, Bcrp1/ABCG2 is expressed in progenitor cells at both ages but is not expressed in neural stem cells derived from the adult.

\section{SP-derived neurospheres display the properties of neural stem cells}

By definition, stem cells are (1) self-renewing and (2) multipotential cells. To confirm that the neurospheres that formed after Hoechst exposure and sorting were derived from neural stem cells, we first tested the SP-derived neurospheres to determine whether they were passageable (i.e., displayed the property of self-renewal). Single spheres derived from both adult and embryonic SP regions were dissociated and plated in the presence of EGF and FGF2. Each of the neurospheres from the SP region (adult or embryonic) gave rise to new spheres $(131 \pm 16.5$ new spheres per single dissociated sphere). Interestingly, when neurospheres derived from embryonic non-SP cells were tested (albeit few in number), $100 \%$ of non-SP-derived single spheres failed to passage ( 0 of 6 ), suggesting that they were derived from progenitor cells rather than neural stem cells (Seaberg and van der Kooy, 2002). Accordingly, the embryonic SP population contains $100 \%$ of all neural stem cells. When adult non-SP neurospheres were passaged, $66 \%$ ( 8 of 12) of neurospheres failed to passage. This suggests that the frequency of a neural stem cell among the adult non-SP cells is 1 in 1045 cells, and therefore the relative enrichment in the SP fraction is $22.8 \times$.

Single neurospheres derived from the SP region were examined for multipotentiality. Immunocytochemistry confirmed that all of the SP-derived spheres contain neurons (MAP2+' ve or 
EMBRYONIC
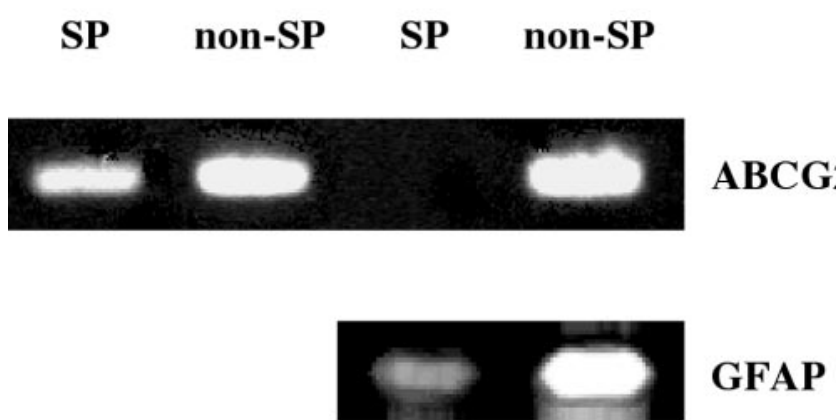

Figure 2. RT-PCR on SP and non-SP fractions. Amplified products of the one-step RT-PCR conducted on RNA isolated from FACS-sorted cells. SP and non-SP fractions from embryonicand adult-derived cells reveal $A B C G 2$ transporter expression in embryonic SP, embryonic nonSP, and adult non-SP cells and the complete absence in adult SP cells. GFAP expression was detected in both SP and non-SP fractions of adult-derived neurosphere cells.

BIII tubulin +'ve) and glia (GFAP+'ve astrocytes and $\mathrm{O} 4{ }^{+}{ }^{\prime}$ ve oligodendrocytes) (Fig. 3).

It has been shown recently that adult forebrain neurospheres are clonally derived from GFAP-expressing cells (Morshead et al., 2003). Neurospheres do not form after the in vivo or in vitro ablation of GFAP-expressing cells, and furthermore, RT-PCR reveals that GFAP is expressed at low levels in whole neurospheres (Morshead et al., 2003). A prediction from these findings is that GFAP expression would be seen in the SP fraction in which the neural stem cells are found. To test this hypothesis we performed RT-PCR on adult SP and non-SP fractions and observed a positive signal from both fractions (Fig. 2). Detection of GFAP expression in the non-SP fraction suggests that (1) non-SP cells express low levels of GFAP or (2) a small subpopulation of non-SP cells express GFAP. Hence, the observation that GFAP is expressed in SP cells is consistent with reports that neural stem cells are GFAP positive; however, detection in the non-SP fraction reveals that GFAP is not a unique marker of neural stem cells.

\section{Further enrichment of neural stem cells}

Rietze et al. (2001) reported the purification of neural stem cells from the primary dissociation of adult brain. Two of the selection criteria were (1) cell size of $>12 \mu \mathrm{m}$ and (2) the low expression of peanut agglutinin. We asked whether using these selection criteria would enrich further the frequency of neural stem cells within the SP region isolated from neurospheres.

A twofold enrichment in neural stem cell isolation was obtained by Rietze et al. (2001) when cells were sorted on the basis of size alone $(>12 \mu \mathrm{m})$; they reported that $80 \%$ of neural stem cells were in the $>12 \mu \mathrm{m}$ diameter population of cells. Because we observed $>80 \%$ of all the neural stem cell activity within the SP region, we sorted the SP on the basis of size to determine whether we could further enrich for the stem cells. A profile from a forward light scatter of neurosphere-derived SP cells revealed that $41 \pm 8 \%$ of the cells were $<10 \mu \mathrm{m}$ in diameter (small) and $23 \pm$ $6 \%$ were $>12 \mu \mathrm{m}$ in diameter (large) (Table 2). Furthermore, $5.5 \%$ of the small diameter cells and $9.7 \%$ of the large diameter cells gave rise to spheres; however, a purity check on these SP fractions (large vs small) before plating revealed a high degree of overlap whereby after the re-sort only $36 \pm 16 \%$ of the small cells remained in this "small" fraction, and $19 \pm 9 \%$ of the cells (originally founding the small fraction) were subsequently found in the large fraction. There was a similar degree of overlap after a purity check of the large cells (Table 2). To test the possibility that the large cells were in fact the result of more than one event (i.e., clumping), we plated large SP cells directly from the FACS analysis into wells of a 24 -well plate at a density of one event per well. The wells were examined $16 \mathrm{hr}$ later to determine the frequency of observing more than one cell per well. We found that $40 \%$ of the wells contained cells (38 of 96), and of these, $47 \%$ had more than one cell per well (18 of 38), suggesting that large cells were likely to be the result of more than one cell per well. Indeed, this finding decreases by $\sim 50 \%$ the frequency that a single "large" cell is a neural stem cell that will form a neurosphere and suggests that the $50 \%$ increase in stem cell frequency within the large cell fraction can be accounted for entirely by clumping during sorting.

The PNA binding criteria reported by Rietze et al. (2001) involved sorting for $\mathrm{PNA}^{\text {lo }}$ cells and resulted in an additional $22 \times$ enrichment in this population. Neurosphere-derived cells from SP populations were sorted further on the basis of their expression of PNA-FITC, and the sorting profile reveals a unimodal distribution of SP-PNA-expressing cells from both the adultand embryonic-derived cell population. We arbitrarily sorted the SP-PNA profile into a small proportion (range 3-17\%) of PNA $^{\text {lo }}$-expressing cells (SP-PNA ${ }^{\text {lo }}$ ) (Fig. $4 a, b$ ) and an SP$\mathrm{PNA}^{\text {hi }}$-expressing population that comprised $36 \%$ of virtually all of the remaining cells within the profile. When the SP-PNA ${ }^{\mathrm{lo}}$ and SP-PNA ${ }^{\text {hi }}$ fractions from adult-derived neurospheres were plated separately, the relative frequency of neurosphere formation did not differ between the two fractions ( $1 \%$ of the cells in both SP-PNA ${ }^{\text {lo }}$ and SP-PNA ${ }^{\text {hi }}$ fractions formed neurospheres). Interestingly, when the SP cells from embryonic-derived neurospheres were sorted into SP-PNA ${ }^{\text {lo }}$ and SP-PNA ${ }^{\text {hi }}$ fractions, $>95 \%$ of the neurospheres were found in the PNA ${ }^{\text {hi }}$-expressing population. Because SP-PNA ${ }^{\text {hi }}$ cells comprise the majority of the SP fraction (range, $65-97 \%$ ), the observation that $>95 \%$ of the stem cells are found here does not represent a true enrichment. Hence, the PNA criteria do not enhance the enrichment of neural stem cells from embryonic or adult neurospheres over that obtained with the SP analysis.

Enrichment for neural stem cells has been described recently using LeX/ssea-1 expression as a criterion for identification (Capela and Temple, 2002). FACS analysis of acutely isolated subventricular zone cells reveals that $4 \%$ of the cells are LeX positive $\left(\mathrm{LeX}^{+}\right)$, and this subpopulation contains the neurosphere-forming cells. Initial examination of neurospherederived cells revealed that $\mathrm{LeX}^{+}$cells are found within the SP and non-SP fractions of embryonic- and adult-derived neurosphere cells (data not shown); therefore, isolation of cells on the basis of LeX expression alone did not enrich for neural stem cells. Accordingly, we set out to determine whether we could further enrich for neural stem cells by sorting for $\mathrm{LeX}^{+}$within the SP fraction. LeX ${ }^{+}$cells represented $48 \%$ (range, 33-68\%) (Fig. $4 d$ ) of all of the embryonic-derived SP cells sorted and gave rise to $80 \%$ of all of the neurospheres that formed $(11.6 \%$ of all cells plated in the $\mathrm{LeX}^{+}$fraction gave rise to neurospheres). This represents a $38 \%$ enrichment compared with sorting for SP alone (1 of 8.5 SP-LeX ${ }^{+}$cells formed a neurosphere vs 1 in 12 cells from SP-only sorting). The adult-derived neurosphere cells revealed a profile whereby $65 \%$ (range, $56-73 \%$ ) of the SP cells were $\mathrm{LeX}^{+}$, and this population gave rise to $72 \%$ of the neurospheres that formed, thereby reflecting only a small $10 \%$ enrichment compared with sorting for SP only. Thus, sorting for $\mathrm{LeX}^{+}$cells within the SP does enrich for neural stem cells from embryonic neurosphere cells. 

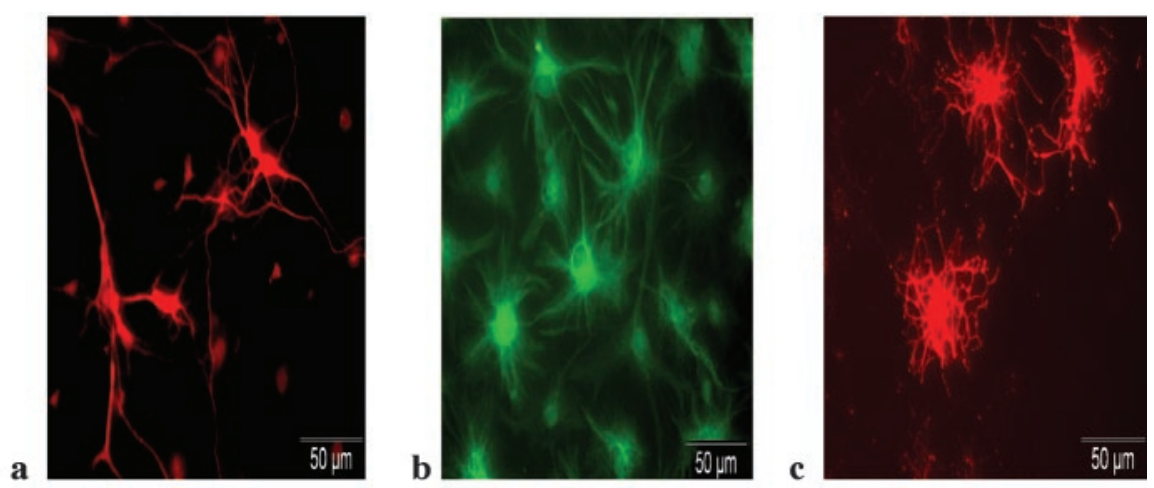

Accordingly, $100 \%$ of the neural stem cells derived from embryonic neurospheres are found within the SP fraction; the non-SP fraction is entirely progenitor cells, and the 278 -fold enrichment is a minimum estimate.

FACS using monoclonal antibodies to cell surface markers also has been reported to enrich for neural stem cells. P75 receptor immunoreactivity prospectively identified putative neural stem cells from the peripheral nervous system (Morrison et al., 1999), and CD133 immunoreactivity allowed isolation of neural stem cells from

Figure 3. SP-derived neurospheres are multipotential. Photomicrographs showing $\beta$ III-tubulin positive neurons (a), GFAPpositive astrocytes $(b)$, and 04-positive oligodendrocytes $(c)$ after $7 \mathrm{~d}$ exposure to differentiation conditions. The photomicrographs are representative of the immunohistochemical staining observed from $100 \%$ of the adult and embryonic SP-derived neurospheres. Scale bars, $50 \mu \mathrm{m}$.

Table 1. Summary chart of enrichment profile for neural stem cells after an SP analysis

\begin{tabular}{lllll}
\hline Source of cells & $\begin{array}{l}\text { SP (as \% of } \\
\text { live cells) }\end{array}$ & $\begin{array}{l}\text { Stem cell } \\
\text { frequency in } \\
\text { SP }\end{array}$ & $\begin{array}{l}\text { Stem cell } \\
\text { frequency in } \\
\text { non-SP }\end{array}$ & $\begin{array}{l}\text { Stem cell } \\
\text { enrichment in SP }\end{array}$ \\
\hline $\begin{array}{c}\text { Embryonic neuro- } \\
\text { spheres }\left(<p 10^{*}\right)\end{array}$ & 3.6 & 1 of 12 & 1 of 3333 & $278 \times$ \\
$\begin{array}{c}\text { Adult neurospheres } \\
\left(<p 10^{*}\right)\end{array}$ & 9.8 & 1 of 46 & 1 of 345 & $7.5 \times$ \\
\hline
\end{tabular}

${ }^{*} p 10=10$ passages in vitro before SP analysis.

Table 2. Sorting on basis of size within the SP fraction of embryonic-derived neurosphere cells

\begin{tabular}{llll}
\hline & $\begin{array}{l}\text { Relative } \\
\text { percentage } \\
\text { within SP }\end{array}$ & $\begin{array}{l}\text { Stem cell } \\
\text { frequency in SP }\end{array}$ & Resort profile $^{b}$ \\
\hline Small ( $<10 \mu$ m diameter) & $41 \pm 8 \%$ & $5.5 \%$ & $\begin{array}{l}\text { Small-36 } \pm 16 \% \\
\text { Large-19 } \pm 9 \%\end{array}$ \\
Large ( $>12 \mu$ m diameter) & $23 \pm 6 \%$ & $9.7 \%$ & $\begin{array}{l}\text { Small- } 17 \pm 6 \% \\
\text { Large-26 } \pm 14 \%\end{array}$ \\
\hline
\end{tabular}

${ }^{a}$ The cell diameters were based on the profile obtained using a standard $3 \mu \mathrm{m}$ bead.

${ }^{b}$ The originally isolated small and large SP fractions were resorted. The resort profiles were similar to the large and small adult-derived SP fractions.

\section{Discussion}

Understanding of the basic biology and therapeutic potential of neural stem cells has been hindered by the inability to prospectively identify neural stem cells and enrich for them. To overcome this barrier we have used a strategy developed for the isolation of hematopoietic stem cells that involves FACS analysis on the basis of the enhanced ability of stem cells to efflux Hoechst dye (Goodell et al., 1996). This dye exclusion paradigm revealed that neural stem and progenitor cells derived from neurospheres exhibit a sorting profile containing a low fluorescing SP that is highly enriched for neural stem cells. Sorting of embryonic-derived neurosphere cells results in an SP region that comprises only $3.6 \%$ of all the live cells yet contains $99 \%$ of all the neurospheres, representing a 278-fold enrichment of neural stem cells. Importantly, the SP cells give rise to neurospheres that are self renewing and multipotent. None of the non-SP-derived neurospheres elicited the cardinal stem cell property of self-renewal (Potten and Loeffler, 1990), and therefore non-SP neurospheres (1\% of all the neurospheres that formed) were derived from progenitor cells and not neural stem cells (Seaberg and van der Kooy, 2002). human fetal brain (Uchida et al., 2000).

Rietze et al. (2001) used an immuno-FACS strategy to isolate adult neural stem cells from primary adult forebrain tissue. The selection criteria were cell diameter $>12$ $\mu \mathrm{m}$, low peanut agglutinin expression, and low heat-stable antigen expression. These criteria resulted in neurosphere formation from 1 to 1.3 of the cells isolated (Rietze et al., 2001). Using the size criteria in an attempt to further enrich for neural stem cells within the SP fraction, we found that sorting on the basis of size criteria is not reliable because the purity of the population is poor, with up to $20 \%$ of the fractions overlapping after reanalysis of sorted populations (compared with $>98 \%$ purity of the SP fraction). The unreliable nature of forward scatter sorting to separate cells on the basis of cell diameter also may explain the discrepancy between Rietze et al. (2001) and the recent findings of Murayama et al. (2002), which reported that SP cells were present only in the small cell fraction isolated from the adult subventricular zone. The size criterion is compromised further by clumping of cells (i.e., more than one cell per event). Sorting for clumps of cells artificially increases the frequency of isolating neural stem cells. Hence, the inability to reliably separate cells on the basis of cell diameter, in combination with the fact that single events are actually representative of more than one cell, makes cell size a weak criterion on which to base sorting procedures.

Perhaps the most significant difference between the enrichment paradigms for isolating neural stem cells is the starting populations of cells: neurosphere-derived cells versus primary dissociated tissue. Using neurosphere-derived cells was advantageous because we could generate millions of cells for sorting, and more importantly, neurospheres are already enriched for neural stem cells (Morshead et al., 2002). Interestingly, Rietze et al. (2001) report that SP sorting of primary adult forebrain tissue is not a viable approach to isolating neural stem cells. In contrast, we did find that the SP from primary tissue was enriched for neural stem cells. Indeed, $100 \%$ of all of the embryonic neural stem cells were found in the SP; however, only 9 of 10,000 cells plated were neural stem cells (our unpublished observations). The lower frequency of neural stem cells within the SP from forebrain dissections may reflect contamination with differentiated cells from the primary dissociation and highlights the prepurification achieved using neurospheres as a starting population (containing only neural stem and progenitor cells).

The PNA ${ }^{\text {lo }}$ criteria for isolating neural stem cells also was investigated using a starting population of neurosphere cells. Rietze et al. (2001) reported an enrichment on the basis of sorting for $\mathrm{PNA}^{\text {lo }}$-expressing cells from primary dissections of adult tissue. We found, however, that regardless of the starting population of neurosphere-derived cells (adult or embryonic), SP cells 
sorted on the basis of PNA expression showed no further enrichment when we compared the relative percentages of SP$\mathrm{PNA}^{\text {lo }}$ and SP-PNA ${ }^{\text {hi }}$ cells that formed neurospheres. Accordingly, the subsequent PNA profile offers no advantage over the SP sort alone. The contradiction between our findings and the findings of Rietze et al. (2001) may reflect the different starting populations; however, it is worth noting that if single events are sometimes the result of more than one cell, then the possibility remains that the $\mathrm{PNA}^{\text {hi }}$ expressing cells (which comprise the vast majority of cells) are contaminating the cultures of Rietze et al. (2001) and giving rise to neurospheres.

We also observed a difference between the embryonic and adult enrichment profiles when examining SP cells on the basis of LeX/ssea-1 expression. $\mathrm{LeX}^{+}$is a carbohydrate moiety highly expressed on pluripotent stem cells (Solter and Knowles, 1978) and has recently been shown to be expressed in adult subventricular zone cells, including stem cells (Capela and Temple, 2002). We observed a further enrichment among SP cells in the LeX ${ }^{+}$ fraction from both embryonic- and adultderived neurosphere cells. The enrichment was enhanced to a greater extent in the embryonic than in the adult SP-LeX ${ }^{+}$ fraction. In general, less neural stem cell enrichment was achieved from adult neurosphere cells than from embryonic neurosphere cells. One possibility is that adult neural stem cells are a less homogenous population of cells relative to embryonic cells or there are age-related differences among stem cell populations. Further support for age-related changes in neural stem cells comes from studies examining the relative frequencies of neurosphereforming cells at various ages of development (ranging from embryonic day 10.5 to adult) using the parameters of cell size, granularity, nestin-enhanced green fluorescent protein expression, Notch1 expression, and cell adhesion molecules such as E-NCAM (Kawaguchi et al., 2001; Cai et al., 2002; Murayama et al., 2002). Similarly, these studies concluded that the characteristics of neural stem cells were not uniform and changed through development.

The substrate responsible for the efflux of Hoechst dye in hematopoietic stem cells is the ABC transporter Bcrp1/ABCG2 (Zhou et al., 2001). Bcrp1/ABCG2 is expressed in a wide variety of stem cells and was suggested as the molecular basis for the SP phenotype isolated from bone marrow and skeletal muscle (Zhou et al., 2001). Contrary to the results from the hematopoietic system, there is no evidence that neural stem cells express Bcrp1/ ABCG2 in the adult. Indeed, the fact that embryonic SP (and not adult SP) expresses the protein further supports the hypothesis that there are age-related changes among neural stem cells. These data suggest that although differential dye efflux can be used to isolate neural stem cells, the protein involved is not Bcrp1/ABCG2. c

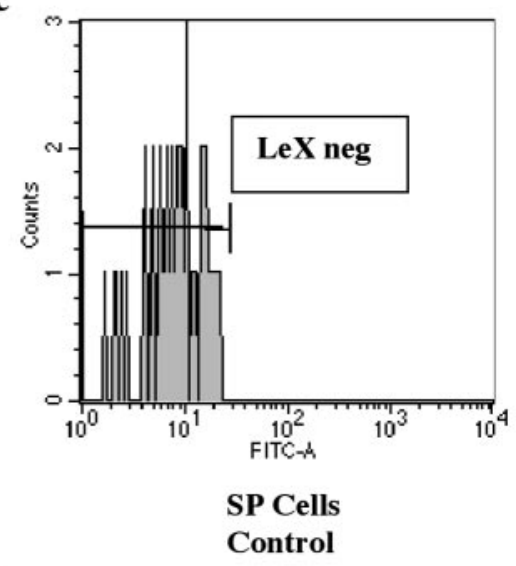

d

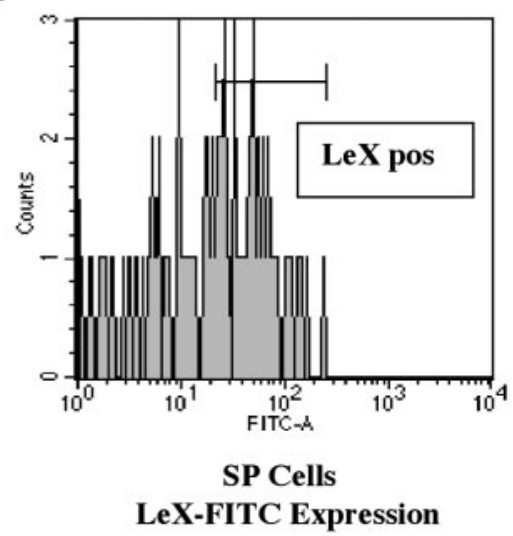

Figure 4. PNA and LeX binding profiles of SP cells. $a$, SP cells were examined before exposure to the PNA-FITC-conjugated antibody to reveal the profile of PNA negative cells. $b$, SP cells were incubated with PNA-FITC antibody and then sorted on the 作 cells (high). The SP-PNA profile shown is from adult neurosphere cells and is not different from that observed with embryonic basis of their FITC fluorescence. The LeX-positive (LeX pos) and -negative fractions were plated separately and assayed for neurosphere formation. Adult and embryonic SP-LeX-FITC had similar unimodal profiles.

It has been shown that GFAP-expressing cells in the adult subventricular zone give rise to neurospheres in vitro (Doetsch et al., 1999; Morshead et al., 2003); therefore, a strong prediction is that the SP fraction will contain GFAP-expressing cells. Indeed, we detected GFAP expression within the SP using RT-PCR. Interestingly, Rietze et al. (2001) were unable to detect GFAP in their isolated neural stem cell population; however, the authors suggest that their method of detection was relatively insensitive (using an adenoviral vector expressing green fluorescent protein under the control of a GFAP promoter). The observation that GFAP is found within the SP fraction is consistent with the fact that neurospheres arise from this fraction; however, GFAP was also detected in the non-SP fraction (composed of progenitor cells) using RT-PCR. Taken together, these results suggest that GFAP is not a good selection marker for neural stem cells.

Overall, the Hoechst dye exclusion protocol is a quick, efficient, and reliable technique to enrich for neural stem cells and, importantly, to obtain a virtually pure population of progenitor cells (the non-SP fraction). Combining the side-population analysis with markers shown recently to enrich for neural stem cells afforded no further enrichment in the case of PNA expression and size criterion (Rietze et al., 2001); however, combining the side-population analysis with LeX expression resulted in a slight 
enrichment over side-population analysis alone. Although the dye exclusion protocol is able to enrich for stem cells from primary tissue, the number of stem cells that are isolated is restrictively low; hence the use of neurospheres as a starting population is beneficial because the neural stem cells have already undergone symmetric divisions during neurosphere formation, thereby increasing their numbers. Furthermore, differential dye efflux can be used to isolate stem cells from various tissues, suggesting that the conserved SP phenotype may represent a common feature of stem cells in general. Interestingly, our data suggest that this common mechanism of dye efflux is mediated by different members of the $\mathrm{ABC}$ transport family in a tissue- and age-dependent manner.

\section{References}

Cai J, Wu Y, Mirua T, Pierce JL, Lucero MT, Albertine KH, Spangrude GJ, Rao MS (2002) Properties of a fetal multipotent neural stem cell (NEP cell). Dev Biol 251:221-240.

Capela A, Temple S (2002) LeX/ssea-1 is expressed by adult mouse CNS stem cells, identifying them as nonependymal. Neuron 35:865-875.

Doetsch F, Caille I, Lim DA, Garcia-Verdugo JM, Alvarez-Buylla A (1999) Subventricular zone astrocytes are neural stem cells in the adult mammalian brain. Cell 97:703-716.

Goodell MA, Brose K, Paradis B, Conner AS, Mulligan RC (1996) Isolation and functional properties of murine hematopoeitic stem cells that are replicating in vivo. J Exp Med 183:1797-1806.

Goodell MA, Rosenzweig M, Kim H, Marks DF, DeMaria M, Paradis G, Grupp SA, Sieff CA, Mulligan RC, Johnson RP (1997) Dye efflux studies suggest that hematopoeitic stem cells expressing low or undetectable levels of CD34 antigen exist in multiple species. Nat Med 3:1337-1345.

Gooi HC, Feizi T, Kapadia A, Knowles BB, Solter D, Evans MJ (1981) Stagespecific embryonic antigen involves alpha 1 goes to 3 fucosylated type 2 blood group chains. Nature 292:156-158.

Kawaguchi A, Miyata T, Sawamoto K, Takashita N, Murayama A, Akamatsu W, Ogawa M, Okabe M, Tano Y, Goldman SA, Okano H (2001) NestinEGFP transgenic mice: visualization of the self-renewal and multipotency of CNS stem cells. Mol Cell Neurosci 17:259-273.

Lendahl U, Zimmerman LB, McKay RD (1990) CNS stem cells express a new class of intermediate filament protein. Cell 60:585-595.

Morrison SJ, White PM, Zock C, Anderson DJ (1999) Prospective identifi- cation, isolation by flow cytometry, and in vivo self-renewal of multipotent mammalian neural crest stem cells. Cell 96:737-749.

Morshead CM, Craig C, van der Kooy D (1998) In vivo clonal analyses reveal the properties of endogenous neural stem cell proliferation in the adult mammalian forebrain. Development 125:2251-2261.

Morshead CM, Benveniste P, Iscove NN, van der Kooy D (2002) Hematopoetic competence is a rare property of neural stem cells that may depend on genetic and epigenetic alterations. Nat Med 8:268-273.

Morshead CM, Garcia AD, Sofroniew M, van der Kooy D (2003) The ablation of glial fibrillary acidic protein-positive cells from the adult central nervous system results in the loss of forebrain neural stem cells but not retinal stem cells. Eur J Neurosci 18:76-84.

Murayama A, Matsuzaki Y, Kawaguchi A, Shimazaki T, Okano H (2002) Flow cytometric analysis of neural stem cells in the developing and adult mouse brain. J Neurosci Res 69:837-847.

Potten CS, Loeffler M (1990) Stem cells: attributes, cycles, spirals, pitfalls and uncertainties. Lessons for and from the crypt. Development 110:1001-1020.

Rietze RL, Valcanis H, Brooker GF, Thomas T, Voss AK, Bartlett PF (2001) Purification of a pluripotent neural stem cell from the adult mouse brain. Nature 412:736-739.

Seaberg RM, van der Kooy D (2002) Adult rodent neurogenic regions: the ventricular subependyma contains neural stem cells, but the dentate gyrus contains restricted progenitors. J Neurosci 22:1784-1793.

Solter D, Knowles BB (1978) Monoclonal antibody defining a stage-specific mouse embryonic antigen (SSEA-1). Proc Natl Acad Sci USA 75:5565-5569.

Storms RW, Goodell MA, Fisher A, Mulligan RC, Smith C (2000) Hoechst dye efflux reveals a novel $\mathrm{CD} 7{ }^{+} \mathrm{CD} 34^{-}$lymphoid progenitor in human umbilical cord blood. Blood 96:2125-2133.

Tropepe V, Sibilia M, Ciruna B, Rossant J, Wagner EF, van der Kooy D (1999) Distinct neural stem cells proliferate in response to EGF and FGF in the developing telencephalon. Dev Biol 208:2251-2261.

Tropepe V, Coles BLK, Chiasson BJ, Horsford DJ, Elia AJ, McInnes RR, van der Kooy D (2000) Retinal stem cells in the adult mammalian eye. Science 287:2032-2036.

Uchida N, Buck DW, He D, Reitsma MJ, Masek m, Phan TV, Tsukamoto AS, Gage FH, Weissman IL (2000) Direct isolation of human central nervous system cells. Proc Natl Acad Sci USA 97:14720-14725.

Zhou S, Schuetz JD, Bunting KD, Colapietro A-M, Sampath J, Morris JJ, Lagutina I, Grosveld GC, Osawa M, Nakauchi H, Sorrentino BP (2001) The $\mathrm{ABC}$ transporter Bcrp1/ABCG2 is expressed in a wide variety of stem cells and is a molecular determinant of the side-population phenotype. Nat Med 7:1028-1034. 